\title{
PERFIL EPIDEMIOLÓGICO DAS OCORRÊNCIAS DE SÍFILIS NO MUNICÍPIO DE NOVO CRUZEIRO-MG - 2010 A 2019
}

\section{EPIDEMIOLOGICAL PROFILE OF SYPHILIS OCCURRENCES IN NOVO CRUZEIRO-MG- 2010 TO 2019}

\author{
Cleuner Barbosa Coelho \\ Graduando em Farmácia Generalista pela Faculdade Presidente Antônio Carlos de \\ Teófilo Otoni. \\ E-mail: cleunerbarbosa@gmail.com \\ Raissa Alves dos Santos \\ Graduando em Farmácia Generalista pela Faculdade Presidente Antônio Carlos de \\ Teófilo Otoni. \\ E-mail: raissajetaime201282@gmail.com \\ Remulo Russel da Cruz Alves \\ Graduando em Farmácia Generalista pela Faculdade Presidente Antônio Carlos de \\ Teófilo Otoni. \\ E-mail: remulorusselcruz@gmail.com \\ Andreia Teixeira Oliveira Santos \\ Doutora em Biotecnologia dos Biocombustíveis. \\ Professora no curso de Farmácia da Faculdade Presidente Antonio Carlos de Teófilo \\ Otoni. Professora no curso de Ciência e Tecnologia da UFVJM \\ E-mail: andreia.compbyte@gmail.com \\ Lucio Onofri \\ Professor - Faculdade Presidente Antônio Carlos UNIPAC, Brasil \\ E-mail: lucioonofri@gmail.com
}

\section{Resumo}

A sífilis é uma doença infectocontagiosa, causada pela bactéria Treponema pallidum, transmitida de forma adquirida (sexualmente), vertical (congênita) e menos comumente de forma indireta (contato com materiais contaminados) ou por hemotransfusão. O diagnóstico é feito por meio de testes treponêmicos ou nãotreponêmicos, a depender do estágio. É uma doença que apresenta tratamento acessível, efetivo e eficaz, mas ainda exibe altas taxas de incidência, representando um desafio para a saúde pública no Brasil. No município de Novo Cruzeiro/MG, tem- 
se observado o aumento da incidência da doença. No tocante aos dados epidemiológicos, considerando o crescente aumento de notificações de sífilis, é de fundamental importância detectar as áreas com maior foco para esses agravos e identificar as barreiras que comprometem o controle da sífilis em nível local, para que se possa adotar medidas de redução dessas infecções e propor medidas de intervenção efetivas. Neste contexto, esta pesquisa objetivou caracterizar a incidência e prevalência da Sífilis no Município de Novo Cruzeiro - MG, entre os anos de 2010 a 2019, a partir de dados coletados no Sistema de Informação de Agravos de Notificação. Trata-se de pesquisa descritiva, com abordagem quantitativa e de coleta retrospectiva em fontes secundárias: Sistema de Informação de Agravos de Notificação (SINAN), excluídas as entradas duplicadas e as de não residentes que tenham sido notificados no município de Novo Cruzeiro, inseridos no Cadastro Nacional de Estabelecimento de Saúde - CNES no 6535488 e Código do IBGE 3145307. O estudo mostrou que foram notificados 39 casos de sífilis entre os anos de 2010 a 2019, com tendência crescente entre os anos de 2015 a 2017, corroborando com os achados nacionais da infecção. O ano de 2017 foi o que apresentou maior numero de notificações de sífilis, com $48 \%$ dos casos. Do total de infectados, a maior parte $(66,6 \%)$ era do sexo masculino. Quanto as gestantes diagnosticadas com sífilis no município, observa-se um diagnostico tardio, sendo durante o parto/curetagem ou pós-parto (nos anos de 2015, 2016 e 2017), indicando fragilidade na assistência prénatal. Somente em 2018 foi que os casos de sífilis congênita foram diagnosticados ainda no pré-natal, em $100 \%$ dos casos. Através deste estudo foi possível perceber que se faz necessário à tomada de medidas preventivas, a fim de reduzir a frequência do numero de casos de sífilis na região.

Palavras-Chave: Prevalência; Sífilis; Assistência Farmacêutica.

\section{Abstract}

Syphilis is an infectious disease, caused by the Treponema pallidum bacteria. It is sexually, congenitally, and less often indirectly transmitted by contact with contaminated objects or by blood transfusion. The diagnosis is done by nontreponemal or treponemal tests, depending on the stage of the disease. It is an illness with an easy, efficient and effective treatment, notwithstanding the high rates of incidence, thus presently posing a serious challenge to public healthcare in Brazil. In the brazilian county of Novo Cruzeiro, an increase in incidence has been observed. As to what concerns the epidemiological data denouncing the sharp rise of syphilis notifications, it is fundamental to detect the most afflicted areas and to identify the hurdles that compromise the control of the disease locally, so that certain measures may be adopted to reduce those infections and to propose effective ways to positively intervene. Within this context, this research aims to quantify the incidence and prevalence of syphilis in the Novo Cruzeiro county of the state of Minas Gerais, between 2010 and 2019, based on data collected by the 'Sistema de Informação de Agravos de Notificação' institution. It is a descriptive research, with a quantitative approach and retrospective gathering of information in secondary sources: Sistema de Informação de Agravos de Notificação (SINAN), excluding all duplicate entries and and 
those by non residents that were notified in the Novo Cruzeiro county, inserted in the 'Cadastro Nacional de Estabelecimento de Saúde - CNES number 6535488' and 'Código do IBGE 3145307'. The study showed that 39 syphilis cases were notified between 2010 and 2019, with a rising tendency between 2015 and 2017, corroborating with the national infection findings. The year of 2017 showed the highest amount of notifications, with $48 \%$ of all cases. The majority of those infected were of the male gender $(66,6 \%)$. Regarding the infected pregnant women of the county, a rather late diagnosis is performed during the delivery or curettage or after the birth of the child (in the years of 2015, 2016 and 2017), clearly indicating that there is a deficiency in prenatal assistance. Only in 2018 were all congenital syphilis cases diagnosed during the prenatal stage in all cases. Through this paper we came to the understanding that preventive solutions have to be found to reduce the frequency of syphilis cases in the region.

Keywords: Prevalence; Syphilis; Pharmaceutical care

\section{Introdução}

A sífilis é uma doença, infectocontagiosa, exclusivamente humana, de evolução crônica, causada pela bactéria Treponema pallidum, uma espiroqueta, flagelada, revestida por envelope externo com três camadas de moléculas de ácido $\mathrm{N}$-acetil murâmico e $\mathrm{N}$-acetil glucosamina. O T. Pallidum é um patógeno exclusivamente humano (TRABULSI, 2009).

A sífilis é transmitida de forma sexual (adquirida) ou vertical (congênita: da mãe para o filho no momento do parto). Em raros casos, ocorre a transmissão indireta (objetos contaminados, perfurocortantes, tatuagens) e hemotransfusão. A penetração do T. pallidum ocorre por fricções ocorridas durante a relação sexual, em que a bactéria atinge posteriormente o sistema linfático. Através da corrente sanguínea atinge outras partes do corpo, podendo afetar diversos órgãos. A resposta imunológica local causa ulcerações e erosões. A resposta sistêmica faz com que ocorra surgimento de complexos imunes que podem ser depositados em diferentes órgãos. A resposta humoral não é capaz de proteger o organismo da bactéria, pois a imunidade celular é tardia, de forma que a bactéria consegue se multiplicar e sobreviver no organismo por longo tempo (AVELLEIRA, 2006).

O diagnóstico é feito com base na fase da doença, por meio de exames diretos (exame em campo escuro, pesquisa direta com material corado), testes imunológicos treponêmicos fluorescent treponemal antibody absorption test (FTA-abs), Enzyme 
Linked ImmunonoSorbent Assay (ELISA), Anticorpo treponêmico hemaglutinação (TPHS) e não treponêmicos (Venereal Disease Research Laboratory -VDRL, Unheated Serum Reagin (USR), Rapid Test Reagin (RPR), Toluidine Red Unheated Serum Test (TRUST).

Na sífilis primária, e em alguns casos de sífilis secundária com lesões, o diagnóstico pode ser feito com prova direta, sendo a sorologia utilizada na segunda ou terceira semana após aparecimento de cancro, onde já é possível detectar anticorpos, conforme determina a Organização Mundial de Saúde (OMS, 2015).

A Sífilis foi incluída na lista de doenças de notificação compulsória no ano de 2005, através da Portaria MS/SVS no. 33, tendo a ficha de investigação liberação para digitação no Sistema Nacional de Agravos de Notificação (SINAN) em 2007. Esta ficha de investigação sofreu variações ao longo dos anos, na busca de uma melhor detecção e acompanhamento do processo de eliminação, assinado em acordos internacionais pelo Ministério da Saúde (BRASIL, 2007).

A título de notificação compulsória, classifica-se pelo Ministério da Saúde, em: sífilis adquirida, sífilis congênita (SC) e sífilis gestacional, sendo a SC de maior destaque para a saúde pública, por produzir maior impacto de morbimortalidade. No entanto, para prevenção da Sífilis Congênita ou Sífilis Gestacional, faz-se necessário a identificação e tratamento precoce da sífilis adquirida (BRASIL, 2010).

Os informes oficiais da Organização Mundial da Saúde (OMS), publicados em fevereiro de 2019 mostram que surgem cerca de 12 milhões de novos casos na população adulta em todo mundo. A maior parte destes casos em países em desenvolvimento. No ano de 2016, havia mais de meio milhão (aproximadamente 661 mil) de casos de sífilis congênita no mundo, resultando em mais de 200 mil natimortos e mortes neonatais, responsáveis por $29 \%$ de óbitos perinatais, $11 \%$ de óbitos neonatais e $26 \%$ de natimortos em todo o mundo (OMS, 2019).

No Brasil, segundo dados da OMS, estima-se que a prevalência média de sífilis adquirida em parturientes varia entre 1,4\% e 2,8\%, com uma taxa de transmissão vertical em torno de $25 \%$ ou seja, 937 mil novos casos de sífilis. A prevalência nas gestantes é de 2,6\%, o que corresponde a quase 50 mil mulheres acometidas por sífilis por ano e 12 mil casos anuais de sífilis congênita (transmitida de mãe para o 
bebê). A taxa de incidência de sífilis congênita é de cerca de quatro casos a cada mil nascidos vivos (OMS, 2019). Em Minas Gerais, foram registrados e notificados no Sistema Nacional de Agravos de Notificação (SINAN), 14 mil casos da doença no ano de 2018 (SINAN, 2018).

Embora a Sífilis seja uma doença curável e de tratamento gratuito ofertado pelo Sistema Único de Saúde (SUS), as notificações de casos têm aumentado a cada ano. A OMS afirma que a erradicação da Sífilis contribuirá para três dos grandes objetivos do milênio: 1) a redução da mortalidade infantil; 2) melhoramento da saúde materna; 3) a luta contra HIV/AIDS dentre outras doenças (OMS, 2019). Diante dos avanços tecnológicos nas ofertas de testes diagnósticos, com expressivas sensibilidades e especificidade, oferta de tratamento eficaz e gratuito e aumento da oferta de profissionais da área de saúde como enfermeiros, farmacêuticos e médicos, a sífilis pode ser facilmente controlada.

Neste contexto, diante do impacto da sífilis na saúde pública e o aumento do número de casos no município de Novo Cruzeiro, é de extrema importância conhecer a realidade da doença na população, para que ações de prevenção e controle sejam adotadas.

\section{Metodologia}

Esta pesquisa trata-se de um estudo quantitativo, de caráter exploratório, com recorte temporal, sendo os dados extraídos do Sistema de Informação de Agravos de Notificação - SINAN/MS nos anos de 2010 a 2019, inseridos no Cadastro Nacional de Estabelecimento de Saúde - CNES, n 6535488 e Código do IBGE 3145307 como município residente de Novo Cruzeiro - MG.

O Município de Novo Cruzeiro/MG possui $1.702,981 \mathrm{~km}^{2}$ de área de unidade territorial e população estimada de 31.331 habitantes estimados para o ano de 2019. No último senso (2010), a população era de 30.725 habitantes (15.469 do sexo feminino e 15.256 do sexo masculino) (IBGE, 2010).

A população de estudo constituiu-se de todos os casos de sífilis notificados no município de Novo Cruzeiro/MG (2010 a 2019), sendo excluídas as notificações 
duplicadas e de pacientes não residentes no município. O município de Novo Cruzeiro localiza-se no estado de Minas Gerais, na região do baixo Jequitinhonha, tendo o IDH de 0,517 , considerado baixo, estando em $27^{\circ}$ lugar entre os menores IDH do estado, entre 853 cidades.

As variáveis investigadas acerca dos pacientes foram: número de casos notificados por ano, gênero, faixa etária, escolaridade, raça/cor, ocupação, possibilidade de gestação.

Os dados obtidos na pesquisa foram dispostos em gráficos e tabelas, visando estabelecer o perfil epidemiológico da sífilis em Novo Cruzeiro e correlacionar com outros estudos, visando estabelecer as possíveis causas da incidência da doença, bem como apontar a contribuição do farmacêutico para a melhora do quadro epidemiológico atual, visto que este profissional participa do diagnóstico e tratamento da doença.

\section{Resultados}

Foram encontrados 39 casos de sífilis no município de Novo Cruzeiro/MG, no período analisado (2010 a 2019) (Tabela 1). O agravo de notificações compulsórias teve inserção de registro no sistema SINAN/MS pelo município de Novo Cruzeiro apenas no ano de 2015, sendo registrados os dados desde o ano de 2010. Observase na Tabela 1 uma taxa de detecção aumentada de 12,6 casos por 100.000 habitantes em 2015, e 59,6 casos por 100.000 habitantes em 2017, e redução para 16 casos por 100.000 habitantes no ano de 2018, apontando um sensível declínio desta patologia. Tais resultados mostram que o município estudado corrobora com os dados nacionais, seguindo a tendência de aumento acentuado do número de casos desde 2010. 
Tabela 1 - Casos e taxa de detecção (por 100.000 habitantes) de sífilis adquirida por ano de diagnóstico. Novo Cruzeiro, Brasil, 2010-2019.

\begin{tabular}{cccccccccccc}
\hline $\begin{array}{c}\text { Sífilis } \\
\text { Adquirid }\end{array}$ & Total & 201 & 201 & 201 & 201 & 201 & 201 & 201 & 201 & 2018 & 2019 \\
a & & 0 & 1 & 2 & 3 & 4 & 5 & 6 & 7 & & \\
\hline $\begin{array}{c}\text { Casos } \\
\begin{array}{c}\text { aaxa de } \\
\text { detecçã } \\
\text { o }\end{array}\end{array}$ & - & 0 & 0 & 0 & 0 & 0 & 4 & 7 & 19 & 5 & 4 \\
\hline
\end{tabular}

Fonte: MS/SVS/SINAN/DCCI - Departamento de Doenças de Condições Crônicas e Infecções Sexualmente Transmissíveis. NOTAS: (1) Dados até 30/03/2020; (2) Dados preliminares para os últimos 05 anos.

Segundo o Ministério da Saúde, esta tendência é global e preocupa a Organização Mundial de Saúde. No Brasil, conforme Tabela 2, o número total de casos notificados entre 2010 a 1019 foi de 650.258 casos de sífilis. Observa-se uma taxa de detecção aumentada de 2,1 casos por 100.000 habitantes no ano de 2010, saltando para 75,8 casos por 100.000 habitantes no ano de 2018. A redução de casos só pôde ser observada no ano de 2019, sendo 32,91 casos por 100.000 habitantes. No estudo de Chiacchio et. al, (2020), a prevalência de sífilis adquirida também se mostrou elevada no Brasil entre os anos de 2010 a 2018 em todas as regiões,com maior prevalência no gênero masculino e com destaque para regiões Sudeste, Norte e Nordeste.

Tabela 2 - Casos e taxa de detecção (por 100.000 habitantes) de sífilis adquirida por ano de diagnóstico. Brasil, 2010-2019

\begin{tabular}{cccccccccccc}
\hline $\begin{array}{c}\text { Sífilis } \\
\text { Adquirida }\end{array}$ & Total & 2010 & 2011 & 2012 & 2013 & 2014 & 2015 & 2016 & 2017 & 2018 & 2019 \\
\hline Casos & 650.25 & 3.92 & 18.31 & 28.08 & 39.56 & 50.87 & 69.72 & 91.72 & 122.67 & 158.05 & 67.30 \\
& 8 & 9 & 7 & 2 & 6 & 6 & 9 & 8 & 9 & 1 & 1 \\
$\begin{array}{c}\text { Taxa de } \\
\text { detecçã } \\
\text { o }\end{array}$ & - & 2,1 & 9,5 & 14,5 & 19,7 & 25,1 & 34,1 & 44,5 & 59,1 & 75,8 & 32,91 \\
\hline
\end{tabular}

Fonte: MS/SVS/SINAN/DCCI - Departamento de Doenças de Condições Crônicas e Infecções Sexualmente Transmissíveis. NOTAS: (1) Dados até 30/03/2020; (2) Dados preliminares para os últimos 05 anos.

Este estudo também apontou uma prevalência de casos diagnosticados no gênero masculino. Em razão do sexo (Gráfico 1), o município de Novo Cruzeiro apresenta elevado número de homens com sífilis, o que corrobora com os estudos de Figueiredo et al., (2011), no qual a concepção de masculinidade e saúde, dificultam a busca de 
tratamento para alguns homens, a partir da concepção errônea que homem não gosta ou não valoriza o cuidado com sua saúde. A maior prevalência no sexo masculino também foi apontada nos estudos de Chiacchio et. al, (2020) e Lupi et al., (2017).

Tais resultados apontam para a necessidade de ampliar a combinação de intervenções de prevenção de doenças sexualmente transmissíveis para homens no Brasil, principalmente os jovens. O monitoramento da prevalência de sífilis e de outras doenças sexualmente transmissíveis, bem como a avaliação do comportamento sexual de risco de jovens do sexo masculino e o nível de conhecimento acerca das formas de transmissão e prevenção são importantes ferramentas para subsidiar a formulação de políticas de prevenção e ações programáticas necessárias ao controle desses agravos.

Outro fator a ser considerada a frente dos resultados obtidos é que a sífilis é um marcador de sexo desprotegido, podendo levar também à transmissão do HIV. No estudo de Flemming e Wasserheit, (1999), este fato foi percebido em estudo com população de rua na cidade de São Francisco, onde os infectados com HIV tinham anteriormente o diagnóstico da doença de sífilis, mostrando-se um significante predito para a infecção pelo HIV.

Gráfico 1: Distribuição percentual de casos de sífilis adquirida por sexo e ano de diagnóstico. Novo Cruzeiro. Brasil, 2010-2019.

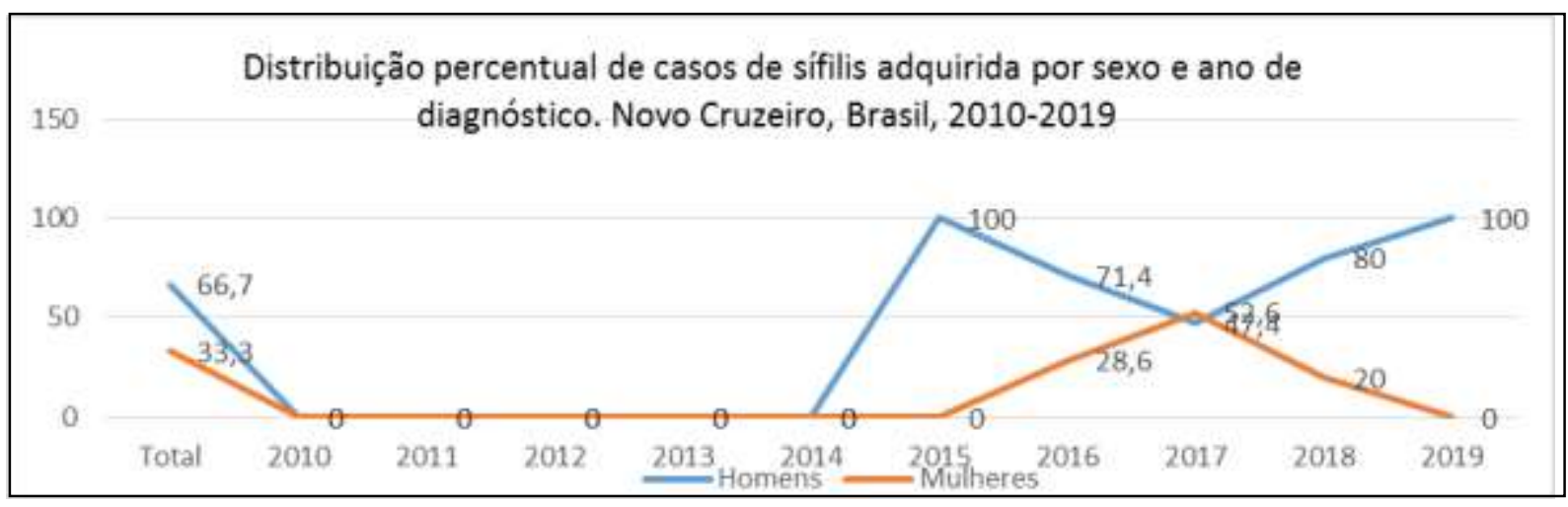

Fonte: MS/SVS/SINAN/DCCI - Departamento de Doenças de Condições Crônicas e Infecções Sexualmente Transmissíveis. NOTAS: (1) Dados até 30/03/2020; (2) Dados preliminares para os últimos 05 anos.

A observação dos dados evidencia claramente que o parceiro sexual é fator preponderante na manutenção da circulação da sífilis no município. A informação das 
consequências da sífilis deve chegar ao conhecimento desta população, como também a sua corresponsabilidade pela importância do diagnóstico e adequado tratamento.

Outro perfil analisado neste estudo foi à sífilis congênita (SC), transmitida por via transplacentária da gestante infectada e não tratada para o recém-nascido. A SC caracteriza-se um grave problema mundial de Saúde Pública, principalmente nos países em desenvolvimento e em muitos países desenvolvidos (SARACENI, 2003; LIMA, et. al., 2006), e continua sendo causa importante de morbidade e mortalidade perinatal.

A SC também é considerada como fator de indicativo de qualidade da assistência à saúde em um determinado local, em virtude da promoção e realização de diagnóstico, do tratamento da gestante e do parceiro na fase de assistência do prénatal, as quais são medidas para promoção e redução da doença (BRASIL, 2007).

De acordo com a Tabela 3, a distribuição percentual de casos de sífilis congênita segundo faixa etária da mãe e por ano de diagnóstico no Brasil entre os anos de 2010 a 2019, destaca-se o ano de 2018, onde a taxa de incidência de sífilis congênita entre as mulheres da faixa etária de 15 a 19 anos, foi de 58,38 mulheres para cada grupo de 1000 mulheres. Esta constante pode ser observada nos anos anteriores (2015, 2016 e 2017). Verifica-se também uma disparidade no tocante à idade em relação aos estudos realizados por Lima (LIMA, et al.,2013), onde $57 \%$ das mulheres se encontravam na faixa etária de 20 a 29 anos, evidenciando uma precocidade na iniciação sexual em relação ao perfil sóciodemográfico das gestantes, representada pelos anos de escolaridade e pela faixa etária. $O$ baixo tempo de escolaridade pode dificultar a adesão ao tratamento e às medidas de prevenção da doença.

$\mathrm{Na}$ gestação, a sífilis produz suas consequências mais nefastas, como abortamentos, partos pré-termos, manifestações congênitas precoces ou tardias e/ou morte do recém- nascido $(\mathrm{RN})$. Pelo fato da sua transmissão vertical, intraútero ou pelo canal do parto, fato no qual, o acompanhamento de pré-natal na rede pública dos municípios é realizado, quase que $100 \%$ por profissionais enfermeiros das unidades básicas de saúde e unidades de saúde da família. 
Tabela 3 - Distribuição percentual de casos de sífilis congênita segundo faixa etária da mãe por ano de diagnóstico. Brasil, 2010-2019.

\begin{tabular}{llllllllllll}
\hline Faixa Etária & Tota & 201 & 201 & 201 & 201 & 201 & 201 & 201 & 201 & 201 & 201 \\
da Mãe & $\mathrm{I}$ & 0 & 1 & 2 & 3 & 4 & 5 & 6 & 7 & 8 & 9 \\
\hline $\mathbf{1 0}$ a 14 anos & - & - & - & - & - & - & - & - & - & - & - \\
$\mathbf{1 5}$ a 19 anos & 66,7 & - & - & - & - & - & 100 & 100 & 100 & 100 & 50 \\
$\mathbf{2 0}$ a 29 anos & 22,2 & - & - & - & - & - & - & - & - & - & 50 \\
$\mathbf{3 0}$ a 39 anos & - & - & - & - & - & - & - & - & - & - & - \\
$\mathbf{4 0}$ anos ou & 11,1 & - & - & 100 & - & - & - & - & - & - & - \\
mais & & & & - & - & - & - & - & - & - & - \\
Ignorado & - & - & - & - & - \\
\hline
\end{tabular}

Fonte: MS/SVS/SINAN/DCCI Departamento de Doenças de Condições Crônicas e Infecções Sexualmente Transmissíveis. NOTAS: (1) Dados até 30/03/2020; (2) Dados preliminares para os últimos 05 anos.

Os resultados expressos na tabela 4 evidencia-se que no ano de 2019, $50 \%$ das mulheres teve o diagnostico pôs parto, e nos anos de 2015, 2016 e 2017, foram $100 \%$ no momento do parto/curetagem, demonstrando a fragilidade da rede de serviço público de saúde, frente as a necessidade de ações estratégicas na oferta de serviços de saúde (pré-natal, triagem sorológica, acesso rápido eficiente de tratamento, busca ativa de parceiros e acompanhamento pós-parto), efetivas no combate à sífilis congênita e suas consequências, e evolução no ano de 2018, notadamente, com o aumento na detecção de sífilis em gestantes, após a ampliação da cobertura de testagem (utilizando a tecnologia de teste rápido - TR) no pré-natal. O teste rápido é um exame treponêmico e a sua utilização na atenção primária possibilita a melhoria da qualidade de diagnóstico no pré-natal, parto e puerpério, possibilitando a adoção de medidas profiláticas da transmissão vertical dos agravos, em virtude da rapidez do diagnóstico e tratamento. 
Tabela 4 - Distribuição percentual de casos de sífilis congênita segundo o momento do diagnóstico da sífilis materna por ano de diagnóstico. Novo Cruzeiro. Brasil, 20102019.

\begin{tabular}{llllllllllll}
\hline $\begin{array}{l}\text { Momento do } \\
\text { diagnóstico da }\end{array}$ & I & 201 & 201 & 201 & 201 & 201 & 201 & 201 & 201 & 201 & 201 \\
sífilis materna & & 1 & 2 & 3 & 4 & 5 & 6 & 7 & 8 & 9 \\
\hline $\begin{array}{l}\text { Durante o pré- } \\
\text { natal }\end{array}$ & 44,4 & - & - & - & - & - & - & - & 50 & 100 & 50 \\
$\begin{array}{l}\text { No momento } \\
\text { do }\end{array}$ & 33,3 & - & - & - & - & - & 100 & 100 & 50 & - & - \\
parto/curetage & & & & & & & & & & & \\
m & & & & & & & & & & & \\
$\begin{array}{l}\text { Após o parto } \\
\text { Não realizado }\end{array}$ & - & - & - & - & - & - & - & - & - & - & - \\
Ignorado & 11,1 & - & - & 100 & - & - & - & - & - & - & - \\
\hline
\end{tabular}

Fonte: MS/SVS/SINAN/DCCl - Departamento de Doenças de Condições Crônicas e Infecções Sexualmente Transmissíveis. NOTAS: (1) Dados até 30/03/2020; (2) Dados preliminares para os últimos 05 anos.

O tratamento farmacológico baseia-se na monoterapia através da penicilina benzatina (MINISTÉRIO DA SAÚDE, 2015). De acordo com Magalhães et al (2011), a não adesão farmacológica da infecção materna na fase inicial possibilita a transmissão vertical par os fetos e por quanto maior distanciamento temporal (sífilis materna tardia) maior o tempo de exposição, maior a frequência de danos ao feto.

E mesmo após tratadas, as mulheres que tiveram sífilis durante a gestação apresentam um risco maior para resultados adversos quando comparadas com mulheres sem história de infecção. Estudos revelam que a probabilidade da infecção fetal é influenciada pelo estágio da sífilis na mãe e pela duração da exposição fetal.

Dessa forma, a transmissão é maior quando a mulher apresenta sífilis primária ou secundária durante a gestação. A infecção fetal provoca entre 30\% a 50\% de morte in útero, parto pré-termo ou morte neonatal. (MINISTÉRIO DA SAÚDE, 2015).

Alguns dos pontos críticos apresentados por este estudo no quesito assistência farmacológica e/ou vigilância epidemiológica foi o percentual de casos de sífilis congênita, segundo esquema de tratamento da mãe por ano de diagnóstico nos anos (2010 - 2019). Tal fato pode ser traduzido na inabilidade do profissional de saúde na captação da gestante, do parceiro e no preenchimento da Ficha de Investigação no 
SINAN, no acompanhamento farmacológico, na inconsistência do banco de dados em virtude da totalidade de tratamentos inadequados/não realizados/ignorados.

Tabela 5 - Distribuição percentual de casos de sífilis congênita segundo esquema de tratamento da mãe por ano de diagnóstico. Novo Cruzeiro. Brasil, 2010-2019.

\begin{tabular}{|c|c|c|c|c|c|c|c|c|c|c|c|}
\hline $\begin{array}{l}\text { Esquema } \\
\text { de } \\
\text { tratamento } \\
\text { materno }\end{array}$ & $\begin{array}{l}\text { Tota } \\
\text { I }\end{array}$ & $\begin{array}{l}201 \\
0\end{array}$ & $\begin{array}{l}201 \\
1\end{array}$ & $\begin{array}{l}201 \\
2\end{array}$ & $\begin{array}{l}201 \\
3\end{array}$ & $\begin{array}{l}201 \\
4\end{array}$ & $\begin{array}{l}201 \\
5\end{array}$ & $\begin{array}{l}201 \\
6\end{array}$ & $\begin{array}{l}201 \\
7\end{array}$ & $\begin{array}{l}201 \\
8\end{array}$ & $\begin{array}{l}201 \\
9\end{array}$ \\
\hline Adequado & - & - & - & - & - & - & - & - & - & - & - \\
\hline $\begin{array}{l}\text { Inadequad } \\
0\end{array}$ & 44,4 & - & - & - & - & - & 100 & - & 50 & 100 & - \\
\hline $\begin{array}{l}\text { Não } \\
\text { Realizado }\end{array}$ & 33,3 & - & - & - & - & - & - & 100 & 50 & - & 50 \\
\hline Ignorado & 22,2 & - & - & 100 & - & - & - & - & - & - & 50 \\
\hline
\end{tabular}

Fonte: MS/SVS/SINAN/DCCI - Departamento de Doenças de Condições Crônicas e Infecções Sexualmente Transmissíveis. NOTAS: (1) Dados até 30/03/2020; (2) Dados preliminares para os últimos 05 anos.

\section{Considerações Finais}

A utilização dos dados legitima a realização de diagnóstico dinâmico da ocorrência de evento em determinado grupo populacional, além de balizar os riscos e identificar a realidade epidemiológica de determinado agravo na referida área geográfica.

Observa-se que os dados do município de Novo Cruzeiro, corrobora com os estudos realizados por Pires et al (2014), em âmbito nacional, o qual confirma que a maioria das infecções pela bactéria ocorrem no sexo feminino, principalmente em virtude de fatores, dentre eles: a falta de informação, acesso limitado aos cuidados de saúde, principalmente no quesito das consultas de pré-natal, gravidez na adolescência, ausência de tratamento do parceiro infectado, desconhecimento dos protocolos e diretrizes terapêuticas no tratamento da sífilis pelos profissionais de saúde, dificuldade na adesão farmacológica dos infectados ao tratamento.

Urge a necessidade do profissional farmacêutico se portar como agente promotor de saúde, com enfoque em sua importância quanto à atenção farmacêutica, cuidado e bem estar do paciente sifilítico, através da adesão ao protocolo de tratamento, na realização dos testes rápidos, na redução da automedicação, na análise da polifarmácia e suas interações medicamentosas bem com os efeitos adversos, na 
sensibilização da população masculina, na promoção da educação sexual, na maior inserção do profissional nos programas de Estratégia de Saúde da Família, nos Programas de Saúde na Escola.

\section{Referências}

AVELLEIRA, João Carlos Regazzi; BOTTINO, Giuliana. Sífilis: diagnóstico, tratamento e controle Syphilis: diagnosis, treatment and control. An Bras Dermatol, v. 81, n. 2, p. 111-26, 2006.

BRASIL, Sífilis Estratégias para Diagnóstico no Brasil, Ministério da Saúde Secretaria de Vigilância em Saúde Departamento de DST, Aids e Hepatites Virais, 2010.

BRASIL. Manual: Testes de Sensibilidade à Penicilina - Brasília: Ministério da Saúde, 1999.

BRASIL. Ministério da Saúde. Secretaria de Vigilância em Saúde. Boletim Epidemiológico - Sífilis. V.47,n. 35-2016.

BRASIL. Ministério da Saúde. Secretaria de Vigilância em Saúde. Departamento de Vigilância Epidemiológica. Boletim Epidemiológico da Sífilis- Ano IV.n.1. Brasília: Ministério da Saúde, 2015.

BRASIL. Ministério da Saúde. Secretaria de Vigilância em Saúde. Departamento de Vigilância Epidemiológica. Boletim Epidemiológico da Sífilis- Ano IV.n.1. Brasília: Ministério da Saúde, 2015.

BRASIL. Ministério da Saúde. Secretaria de Vigilância em Saúde. Departamento de DST/AIDS e Hepatites Virais. Teste Rápido HIV e Sífilis. Rede Cegonha, 2010.

BRASIL. Ministério da Saúde. Secretaria de Vigilância em Saúde. Departamento de Vigilância, Prevenção e Controle das DST, Aids e Hepatites Virais. Agenda de Ações Estratégicas para Redução da Sífilis Congênita no Brasil. Brasília -DF, 2017.

BRASIL. Ministério da Saúde. Secretaria de Vigilância em Saúde. Departamento de Vigilância Epidemiológica. Situação Atual da Sífilis no Brasil e de Penicilina Benzatina para prevenção da Sífilis Congênita. Brasília, 29 de setembro de 2015.

BRASIL. Plano Nacional de Saúde - 2016 - 2019. BRASILIA- DF, 2016.

BRASIL. Protocolo Clínico e Diretrizes Terapêuticas Infecções Sexualmente Transmissíveis - Brasília: Ministério da Saúde, 2015. 
BRASIL. Protocolo Clínico e Diretrizes Terapêuticas para Atenção Integral às Pessoas com Infecções Sexualmente Transmissíveis. Brasília - DF, 2016.

BRASIL. Protocolo de Investigação de Transmissão Vertical. Ministério da Saúde,2016.

BRASIL. Transmissão Vertical do HIV e Sífilis: Estratégias para redução e eliminação - Brasília: Ministério da Saúde, 2014.

BRASIL.GM. Portaria no 3.275, de 26 de dezembro de 2013. Altera a Portaria no 77/GM/MS, de 12 de janeiro de 2012, que dispõe sobre a realização de testes rápidos, na atenção básica, para a detecção de HIV e sífilis, assim como testes rápidos para outros agravos, no âmbito da atenção pré-natal para gestantes e suas parcerias sexuais. Disponível em:

http://bvsms.saude.gov.br/bvs/saudelegis/gm/2013/prt3275_26_12_2013.html. Acesso em: 11 mar. 2020.

CHIACCHIO, AD; ESCOBAR, ND; GILO, NF; BEDRAN, SC; PRIEB, A; SOUSA, MTB. Perfil Epidemiológico de sífilis adquirida nas regiões do Brasil no período de 2010 a 2019. Revista Amazônia Science \& Health. 2020. Vol. 8. № 2.

DATASUS. Ministério da Saúde. Tecnologia da Informação a Serviço do SUS. Disponível em: http://tabnet.datasus.gov.br/cgi/tabnet.exe?pacto/2012/cnv/pactgo. def. Acesso em: 30 mar. 2020.

FIGUEIREDO, W.S.; SCHRAIBER, L.B. Concepções de gênero de homens usuários e profissionais de saúde de serviços de atenção primária e os possíveis impactos na saúde da população masculina. Cienc. Saúde Coletiva, 2011, v.16, suppl.1, p.935-944. Disponível em: Acesso em: 10 fev. 2020.

FLEMMING D, WASSERHEIT J. From epidemiological synergy to public health policy and practice: the contribution of other sexually transmitted diseases to sexual transmission of HIV infection. Sex Transm Infect 1999; 75: 3-17.

IBGE - Instituto Brasileiro de Geografia e Estatística. Censo demográfico. Minas Gerais. Código do Município 3145307. Estimativas da população. Disponível em: https://cidades.ibge.gov.br/brasil/mg/novo-cruzeiro/panorama. Acesso em: 13 jun. 2020.

LIMA, LHM; GURGEL, MFC; MOREIRA-SILVA, SF. Avaliação da sífilis congênita no Estado do Espírito Santo. DST - Jornal Brasileiro de Doenças Sexualmente Transmissíveis. 2006; 18(2):113-116. 
LIMA, Marina Guimarães et al. Incidência e fatores de risco para sífilis congênita em Belo Horizonte, Minas Gerais, 2001-2008. Ciência \& Saúde Coletiva, v. 18, p. 499506, 2013.

MAGALHÃES, D.M.S.; KAWAGUCHI, I.A.L.; DIAS, I.; CALDERON, I.M.P. A sífilis na gestação e sua influência na morbimortalidade materno-infantil. Comunicação em Ciências da Saúde, Brasília, v.22, n.1, p.43-54, 2011.

PIRES, A; OLIVEIRA, D; ROCHA, G; et al. Ocorrência de sífilis congênita e os principais fatores relacionados aos índices de transmissão da doença no Brasil da atualidade. Revista Uningá. Vol. 19, n.1, pp. 58-64. 2014.

SARACENI V. Leal MC. Avaliação da efetividade das campanhas para eliminação da sífilis congênita na redução da morbi-mortalidade perinatal. Município do Rio de Janeiro, 1999-2000. Cadernos de Saúde Pública 2003; 19(5): 1341-1349.

SINAN. Sistema de Informação de Agravos de Notificação. Secretaria de Saúde do Estado de Minas Gerais. Novo Cruzeiro, 2020.

TRABULSI, L. R.; ALTERTHUM, F. Microbiologia. 5.ed. São Paulo: Atheneu, 2008.

VIGILÂNCIA EPIDEMIOLÓGICA. Boletim Epidemiológico da Sífilis- Ano IV.n.1. Brasília: Ministério da Saúde, 2015. 